Sir,

\section{Reply to Dr Haigis}

Dr Haigis' comment about the nomenclature is very justified. About $\mathrm{ACD}=\mathrm{AtoACD}(\mathrm{ELPtoA}($ ELPconst)), this function converts ELP constant to ACD constant for the particular IOL. As can be seen in the definition of the HofferQ function in the appendix, ACD is first defined as a variable of the double type and is assigned the value of ACD constant (personalised ACD constant or manufacturer's ACD constant, whatever) in the first step; then this value is modified as we go down the next steps inside the function. Unfortunately, the whole subject of biometry is replete with overlapping and sometimes confusing terms, for example, ACD (is it ac depth or ELP?), corneal height (from corneal vertex or from secondary principal plane or posterior surface?), $K$ value (optical or keratometric?), axial length (ultrasonic or optical?), etc.

About the computation of the predicted ACD according to Dr Hoffer's 1993 paper, in my article I did mention that I already experimented with all these data and it gave identical values of the predicted ACD $(4.40887,3.14482$, and 6.06225 in examples 1,2 , and 3, respectively). I think our confusion is from the supposition of ELP constant being equal to ACD constant, which it is not.

\section{S Basu}

Department of Ophthalmology, Apollo Gleneagles Hospital, 58, Canal Circular Road, Kolkata 700054, India

Correspondence: S Basu,

Tel: + 91332320 3040;

Fax: + 913323205218.

E-mail: sbasu2003@doctors.net.uk

Eye (2007) 21, 550. doi:10.1038/sj.eye.6702560; published online 29 September 2006

Please note that this article is linked to the following Correspondence articles, which were published in the March 2007 issue of Eye (issue 21.3):

Hoffer KJ. Errors in self-programming the Hoffer Q formula. Eye 2007; 21: 429. doi:10.1038/sj.eye.6702559.

Basu S. Reply to Dr Hoffer. Eye 2007; 21: 430. doi:10.1038/ sj.eye.6702561.

Haigis W. Reply to Basu S et al. Eye 2007; 21: 430-431. doi:10.1038/sj.eye.6702562.
Sir,

Reply to letter from Cooke et al: silicone oil migration causing increasing proptosis $\mathbf{1 3}$ years after retinal surgery

This letter purports to attribute the late complications of unsuccessful surgery for the repair of retinal detachment due to trauma, to silicone oil. The eye in question is certainly unsightly, but there is no evidence from the report that the condition was progressive as implied in the title. The eye was said to have been more prominent only for 6 months, not progressively so. The title implies that the proptosis was due to the silicone; yet the CT scan shows a mass to the medial side of the eye, with limited spread more posteriorly. Had the proptosis been related to the extraocular movement to the silicone, there would have been evidence of a mass behind the eye.

The eye was reported as showing axial myopia as evidenced by an A-scan measurement. The authors should surely have known that ultrasound measurements in the presence of silicone oil always show an abnormal axial length owing to attenuation of the sound signal through the silicone. However, the CT scan does show an enlarged eye with some lateral displacement owing to a medially situated mass. It is highly probable that this previously traumatised eye had shown progressive enlargement due to glaucoma complicating unsuccessfully treated retinal detachment as evidenced by the appearance of the cornea. This would have led to a high risk of exposure and an unsightly eye. It is noteworthy that there appeared to be no history of pain from this eye.

It is also noteworthy that the histopathology showed only a relatively mild inflammatory reaction. This therefore could not be regarded as a granuloma. This term should be restricted to a mass involving chronic inflammation.

Silicone oil may leak from a glaucomatous eye, such as the one described in this letter. It is the result of scleral rupture, usually at one of the parsplanar sclerostomies used for vitrectomy. We have seen this in some patients where oil droplets leak slowly beneath the conjunctiva. It does not however cause such a red and unsightly eye unless other problems are present. In this case, it is highly likely to have been the result of corneal exposure and not oil 'granuloma'.

The analogy with historic techniques for breast augmentation has been used by critics of the use of silicone oil for the treatment of complex retinal detachment. The comment in this letter begins with the statement that oil granuloma occurs when bulky mineral oils are injected into body tissues. It is true that serious problems did occur with the use of mineral oil for breast 